\title{
Diffusion Weighted and Dynamic Contrast Enhanced Magnetic Resonance Imaging in Carcinoma of Cervix: Role of Apparent Diffusion Coefficient Value and Time Intensity Curve Pattern in Chemoradiotherapeutic Response Evaluation
}

\section{Raghavendra H K¹, Alpana Manchanda², Anju Garg ${ }^{3}$, Kishore Singh", Gauri Gandhi ${ }^{5}$}

\author{
${ }^{1}$ Consultant Radiologist, Satyakiran Healthcare, Atlas Road, Sonipat- 131001, India \\ ${ }^{2}$ Director Professor, Department Of Radiodiagnosis, Maulana Azad Medical College, Bahadur Shah Zafar Marg, \\ New Delhi- 110002, India \\ ${ }^{3}$ Director Professor And Head Of Department, Department Of Radiodiagnosis, Maulana Azad Medical College, \\ New Delhi, India. \\ ${ }^{4}$ Director Professor And Head Of Department, Department Of Radiotherapy, Maulana Azad Medical College, \\ New Delhi, India. \\ ${ }^{5}$ Director Professor, Department Of Obstetrics And Gynecology, Maulana Azad Medical College, New Delhi, \\ India.
}

Corresponding Author: Raghavendra H K

DOI: https://doi.org/10.52403/ijrr.20220103

\begin{abstract}
Introduction: Magnetic resonance imaging (MRI) is a widely used imaging modality in the imaging evaluation of carcinoma of cervix. The aim of our study was to evaluate the response in carcinoma cervix patients following chemoradiotherapy by Diffusion weighted (DW-MRI) and Dynamic contrast enhancedMRI (DCE-MRI).

Methods: 21 inoperable biopsy proven patients (mean age 48.43 years) of carcinoma cervix were included in the study. All patients underwent MRI (conventional, DW and DCE) of the pelvis thrice. Baseline MRI, Post chemotherapy MRI after neoadjuvant chemotherapy and Post chemoradiotherapy MRI after completion of concurrent chemoradiotherapy. Post treatment apparent diffusion coefficient (ADC) values and Time intensity curve (TIC) pattern were compared with baseline values.
\end{abstract}

Results: Baseline mean ADC value of all patients was $0.82 \times 10^{-3} \mathrm{~mm}^{2} / \mathrm{s}$. After completion of treatment, 18 patients showed complete resolution of tumor and showed $0.50 \times 10^{-3}$ $\mathrm{mm}^{2} / \mathrm{s}$ increase in mean ADC value from baseline MRI which was significantly higher than remaining 3 patients with residual tumor $\left(0.50 \times 10^{-3} \mathrm{~mm}^{2} / \mathrm{s} \mathrm{v} / \mathrm{s} 0.17 \times 10^{-3} \mathrm{~mm}^{2} / \mathrm{s}\right)$. ADC threshold value of $1.15 \times 10^{-3} \mathrm{~mm}^{2} / \mathrm{s}$ was defined, differentiating the residual tumor from the healthy cervical tissue after chemoradiation. On post treatment MRI, 17 out of 18 patients with complete resolution of tumor showed increasing trend of enhancement on TIC and only one patient showed plateau pattern. 2 of the 3 patients with residual tumor showed washout pattern and one patient showed plateau pattern.

Conclusion: ADC values and TIC pattern differ in patients with complete response to chemoradiotherapy from patients with residual tumor, so helps in differentiating residual tumor from cancer free cervix.

Keywords: Carcinoma of cervix;

Chemoradiotherapy; Diffusion weighted MRI;

Dynamic contrast enhanced MRI; Time intensity curve. 


\section{INTRODUCTION}

Cervical cancer is the $4^{\text {th }}$ most common cancer in women in the world for both incidence and mortality as per the latest data from global cancer statistics 2018. ${ }^{[1]}$ Accurate tumor staging is essential for optimal treatment planning and prognosis. Cervical cancer is staged by clinical examination according to the International Federation of Gynecology and Obstetrics (FIGO) staging system. According to the new FIGO staging (2018) it is recommended that imaging to be used when available, to supplement clinical findings with respect to tumor size and extent. ${ }^{\text {[2] }}$

Magnetic resonance imaging (MRI) has evolved, and is now a widely used imaging modality in the initial staging of these tumors. ${ }^{[3,4]}$ Conventional MRI has limitations when the morphological appearance of early recurrent or residual disease overlaps with normal pelvic anatomy or treatment effects in the pelvis. Imaging within three months of therapy is particularly challenging to interpret as the tumour microenvironment is affected by hypoxia, granulation tissue and oedema and both residual disease and radiation fibrosis show increased T2 signal intensity. [5] Previous studies which have correlated the post treatment MRI results with histopathological findings in patients with cervical cancer have shown that conventional MRI is limited by false positive results with a sensitivity and specificity of around $80 \%$ and $55 \%$ respectively for the detection of residual disease. ${ }^{[6]}$ Diffusion Weighted-MRI (DWMRI) and Dynamic contrast enhanced-MRI (DCE-MRI) can be used to overcome these limitations and to predict early therapeutic response.

Diffusion Weighted-MRI is a functional imaging technique, wherein the image contrast is derived from inherent difference in restriction of the movement of water molecules. ${ }^{[7]}$ Quantitative assessment of the tumor microenviroment can be derived from the apparent diffusion coefficient (ADC) which is generated from combining serial b-values. The mean ADC of cervical cancer is known to increase during and after chemoradiotherapy such that early alterations in ADC can be used to predict early therapeutic response. [8,9] Hence, DWI can be used to assess changes in tumor cellularity over time in response to treatment. ${ }^{[10,11,12]}$

Dynamic Contrast Enhanced-MRI can be used in the assessment of tissue perfusion and oxygenation within the tumour microenvironment by using gadolinium based contrast agents. The ability to evaluate the extent of hypoxia may be a useful predictive tool in identifying which patients may benefit from additional or more aggressive therapies. Imaging attention has been centered towards further evaluating DCE-MRI as a predictive perfusion biomarker Chemoradiation has been found to be more effective in tumors with higher tissue permeability on dynamic MR images than in those with lower tissue permeability, resulting in improved local control. ${ }^{[13,14,15]}$

The present study was undertaken to evaluate the morphological and functional imaging features of carcinoma cervix and response evaluation following chemoradiotherapy using conventional MRI and functional MRI (DW and DCE-MRI).

\section{MATERIALS AND METHODS 2.1. Patients}

This prospective study was conducted after obtaining approval and ethical clearance from the Institutional Ethical Committee. Informed consent was taken by all patients.

21 inoperable biopsy proven patients of carcinoma cervix (mean age of 48.43 years, age range 32-67 years) were evaluated between October 2016 to September 2018. Revised FIGO 2018 guidelines were used to stage the tumor. Of the total 21 patients evaluated, 12 were clinical FIGO stage IIB, 8 were IIIB and 1 patient was of stage IIIA. 


\subsection{MRI protocol}

All the patients underwent MRI of the pelvis thrice $.1^{\text {st }}$ (Baseline) MRI, $2^{\text {nd }}$ (Post chemotherapy) MRI after 2 cycles of neoadjuvant chemotherapy and $3^{\text {rd }}$ (post chemoradiotherapy) MRI after 6-8 weeks of completion of 8 weeks of concurrent chemoradiotherapy (Figure 1).

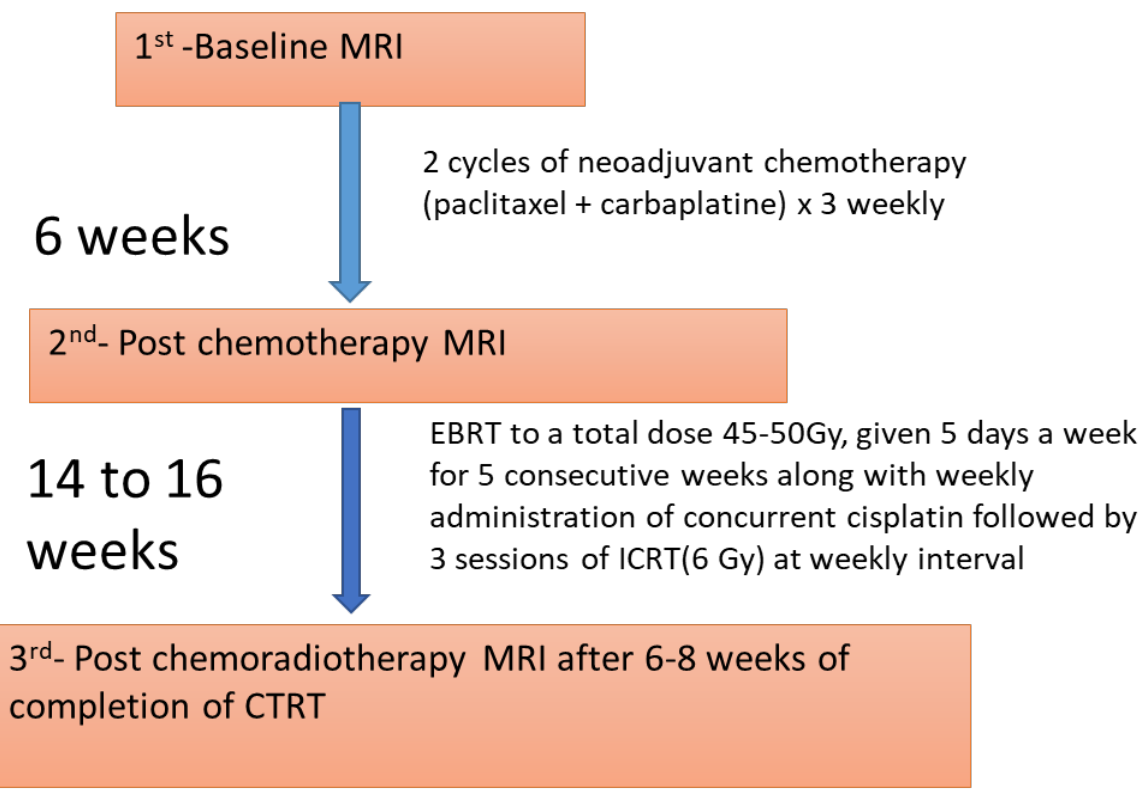

Figure 1: Treatment protocol and MRI timing study of patients

Multiplanar magnetic resonance imaging of the pelvis was done on a 3 Tesla MR Scanner (Magnetom Skyra, Siemens), using phased array body coil. The conventional MR sequences acquired included: axial and sagittal fast spin-echo (FSE) T2-weighted sequences and axial FSE T1-weighted sequences. Sagittal images were acquired parallel to the endocervical canal and Axial scans were planned perpendicular to endocervical canal. The parameters used were as follows: T2 weighted sequences (TR-5000 to 5200 $\mathrm{ms}$, TE-97 ms), T1 weighted sequences (TR- $580 \mathrm{~ms}$, TE- $20 \mathrm{~ms}$ ), Field of view (FOV) $270 \mathrm{~mm}$ to $300 \mathrm{~mm}$ and slice thickness of $3 \mathrm{~mm}$.

After acquiring the conventional MR sequences of the pelvis, DW-MRI was performed in the axial plane using a single shot echo-planar diffusion weighted sequence (TR- $4400 \mathrm{~ms}$, TE- $69 \mathrm{~ms}$ ) without breath hold at b values of 50, 400 and 800 $\mathrm{s} / \mathrm{mm}^{2}$ with generation of ADC maps.
DCE-MRI was then performed. 0.1 $\mathrm{mmol} / \mathrm{kg}$ body weight of gadolinium was injected with a pressure injector at a rate of $2 \mathrm{ml} / \mathrm{sec}$ followed by a bolus of $20 \mathrm{ml}$ saline flush. 3D T1W volume interpolated breathhold examination (VIBE) sequences were used to acquire DCE-MR images through the cervical region. Actual imaging time for a single dynamic acquisition was 30 seconds, and six acquisitions were obtained in the axial plane (1 before contrast injection and 5 after contrast injection) to evaluate the contrast enhancement pattern. Delayed T1W fat suppressed contrast enhanced scans in axial and sagittal planes were obtained three minutes after contrast administration.

\subsection{Image analysis}

Three readers with different experience levels in gynecological MRI (Reader 1, 3 year; Reader 2, 12 years; and Reader 3, 15years) independently reviewed 
all sequences and formed a consensus interpretation of all scans.

Conventional MR images were analysed for tumor size, signal intensity characteristics on $\mathrm{T} 1$ and $\mathrm{T} 2$ weighted sequences, local extension, and lymph node status. FIGO staging of tumor was assigned based on imaging findings. Lymph nodes of size $\geq 10 \mathrm{~mm}$ in short axis were considered as involved.

Diffusion weighted MR images were analysed and region of the cervical carcinoma and enlarged lymph nodes which showed increased signal intensity on high 'b' values ( $\left.b=800 \mathrm{sec} / \mathrm{mm}^{2}\right)$ with decrease in signal in the corresponding ADC map were considered as showing restricted diffusion. Multiple (three) quantitative ADC values were obtained by placing ROI over the lesion avoiding necrotic areas and a mean ADC value was finally obtained. Axial sections were used for ADC value calculation.

DCE-MR images were used to generate time-signal intensity curves (TIC) using the mean curve software package (Siemens) by placing ROI over the enhancing component of the cervical mass. In patients with complete resolution of the tumor, TICs was generated by placing ROI over the cervical stroma. 3 patterns of TICs were observed i.e., progressive enhancement, initial uptake followed by plateau phase towards the later part of the study and initial uptake with washout towards the later part of the study.

On baseline MRI tumor size, signal characteristics, extension to surrounding structures, ADC values, and TIC pattern recorded (Figure 2).
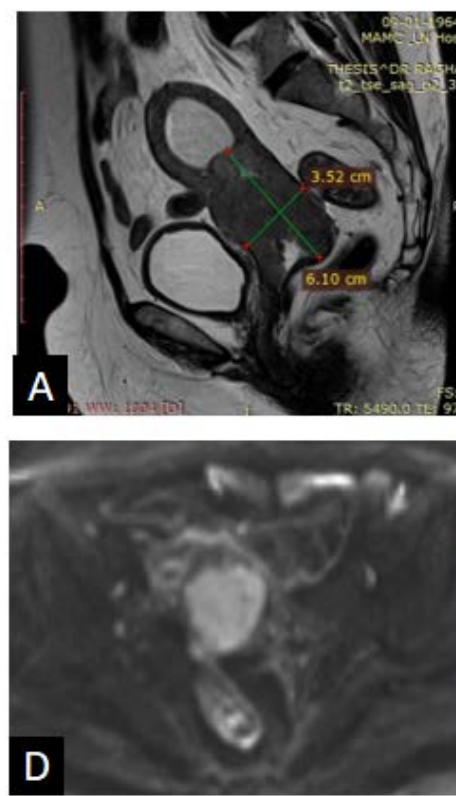
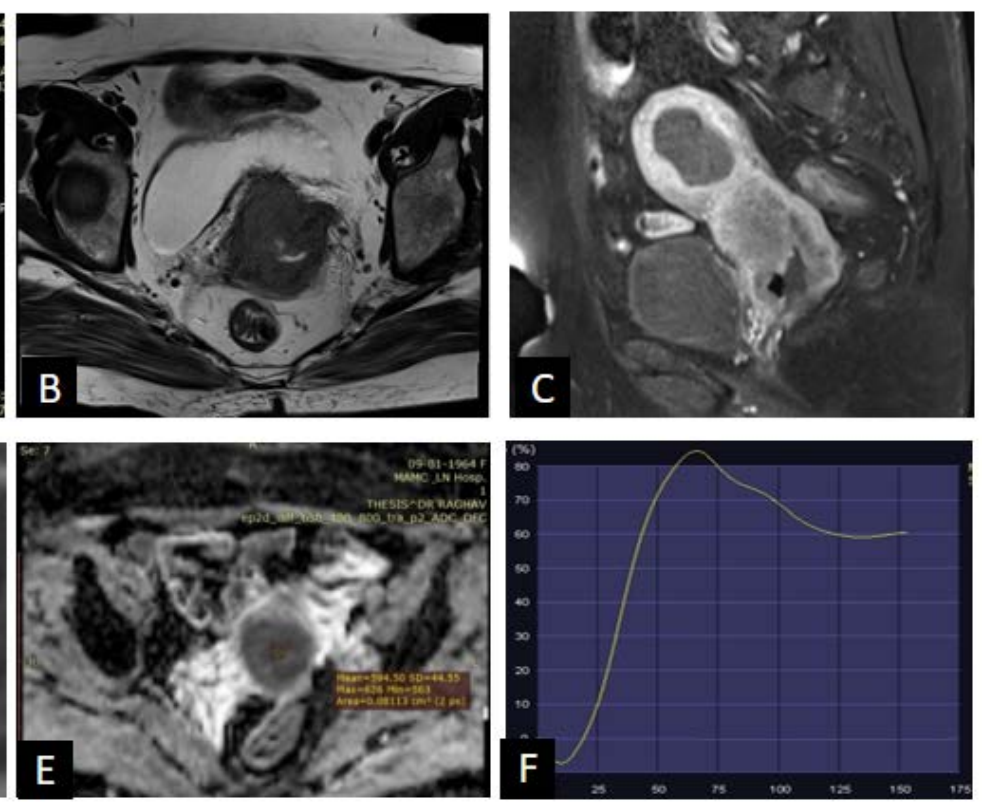

Figure 2: Baseline MRI in a 52 year female

Baseline MRI (A-F): Sagittal T2W (A) and axial T2W(B) MR images of pelvis show infiltrating hyperintense lesion in cervix extending to upper vagina and bilateral parametrium. The lesion shows heterogeneous enhancement on post contrast scan (C). Axial DW image (D) and corresponding ADC map (E) depicts cervical lesion to be bright on DWI and dark on ADC map suggesting diffusion restriction with mean ADC value of $0.59 \times$
$10^{-3} \mathrm{~mm}^{2} / \mathrm{s}$. TIC(F) obtained by placing ROI over the lesion shows initial uptake followed by washout towards later part of the study.

On post chemotherapy MRI therapeutic response was assessed based on change in size of tumor, ADC values and enhancement pattern compared to baseline MRI (Figure 3). 

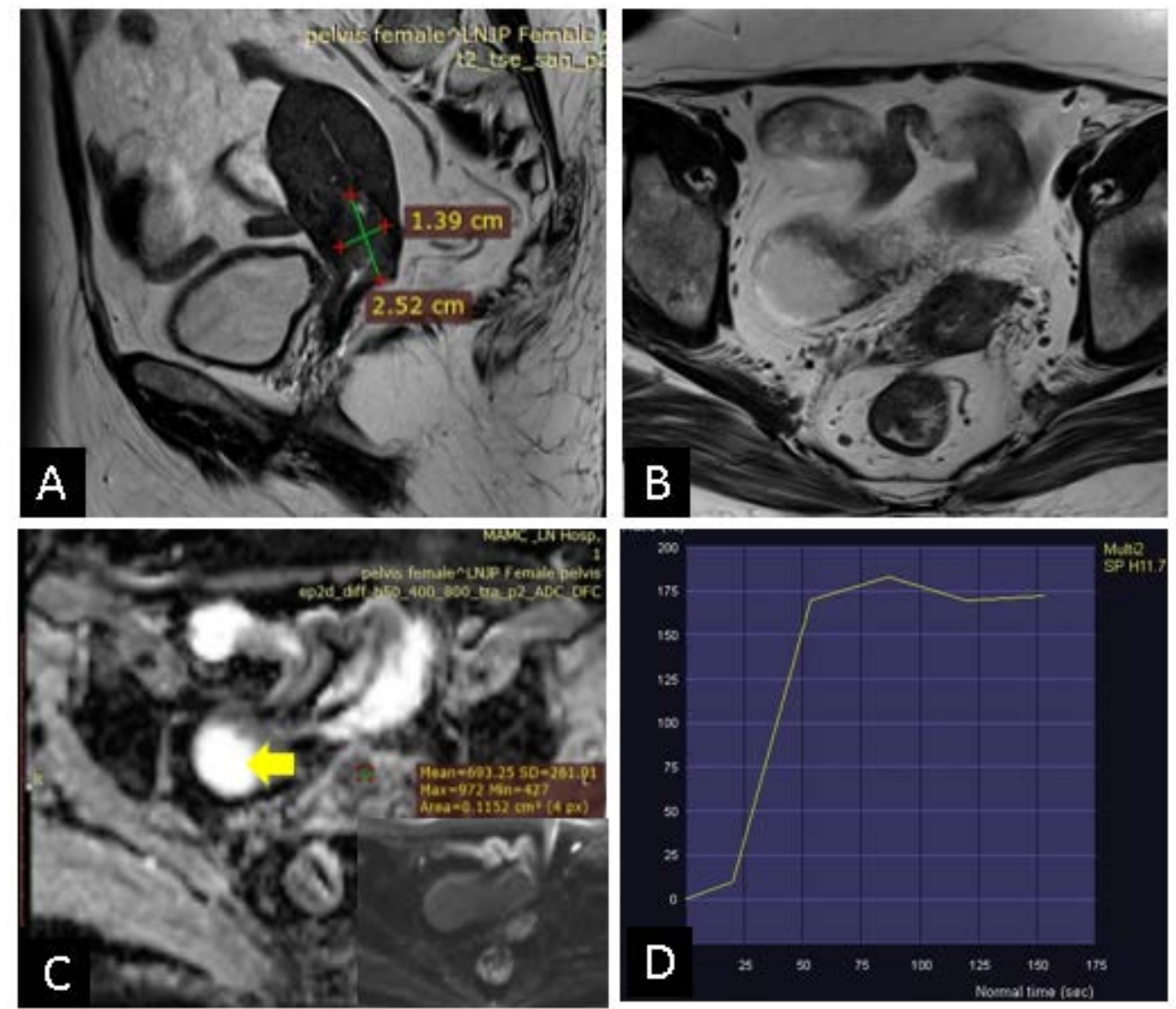

Figure 3:Post chemotherapy MRI

Post chemotherapy MRI (A-D): T2W sagittal (A) \& axial(B) image shows reduction in size of the cervical lesion from baseline scan. The lesion shows diffusion restriction (arrow) on ADC map (C) with increase in ADC value to $0.69 \times 10^{-3} \mathrm{~mm}^{2} / \mathrm{s}$ from baseline MRI. TIC(D) shows plateau pattern of enhancement.
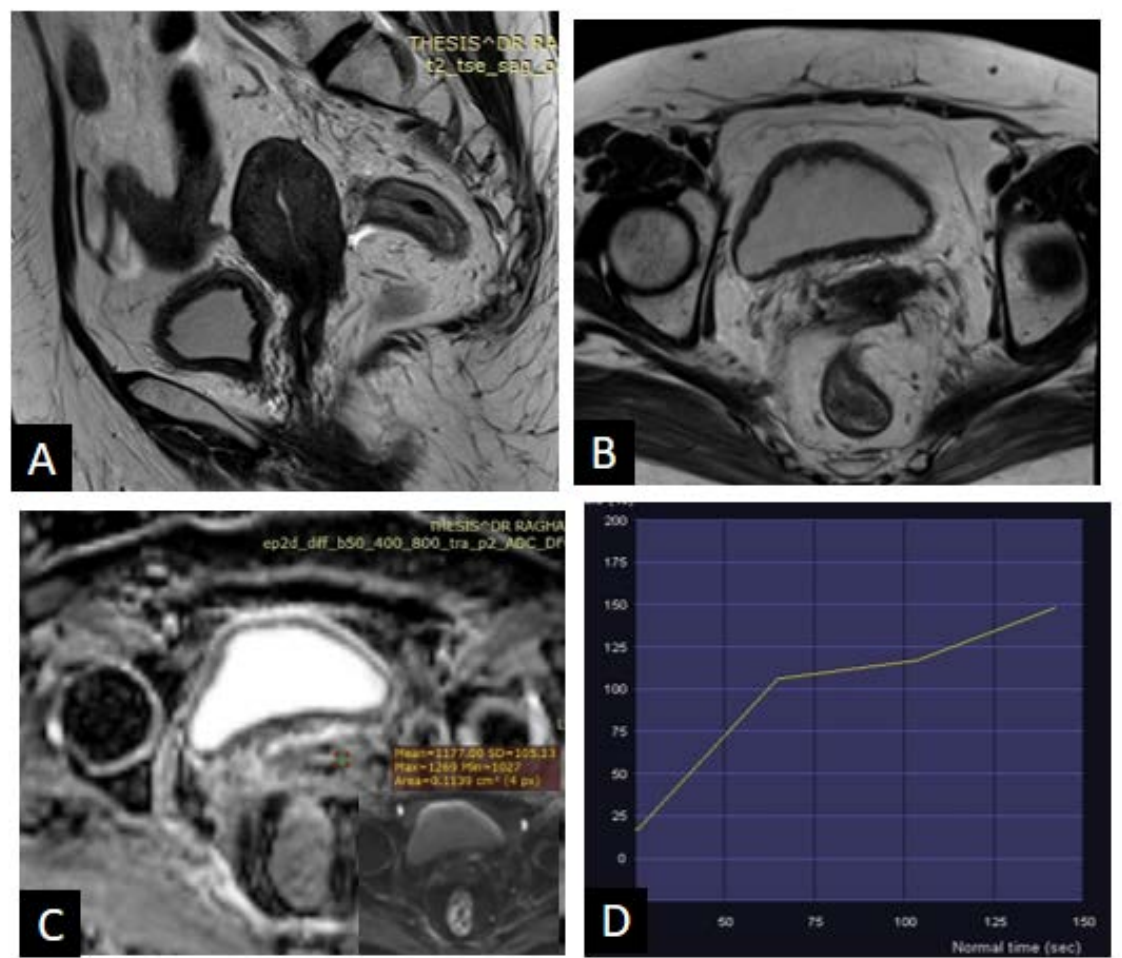

Figure 4: Post chemoradiotherapy MRI 
Post CTRT MRI was analysed for any residual mass lesion. T2 hyperintense lesion showing diffusion restriction was considered as residual mass instead of just T2 hyperintensity as T2 hyperintensity can be present due to post radiotherapy changes. If residual mass lesion was present; its size, extent, ADC value and enhancement pattern were noted and compared with previous MRI scans. If residual lesion was not seen, then ADC values and enhancement pattern of cervical stroma in the region of tumor prior to treatment was obtained for comparison with the previous two MRI scans (Figure 4 and 5).

Post CTRT MRI(A-D): T2 W Sagittal (A) \& axial (B) images reveal complete resolution of tumor. No restricted diffusion noted in cervix with further increase in mean ADC value to $1.12 \times 10^{-3} \mathrm{~mm}^{2} / \mathrm{s}$ from previous MRI scans(C). ROI over cervical stroma shows progressive enhancement with no washout in the TIC (D).
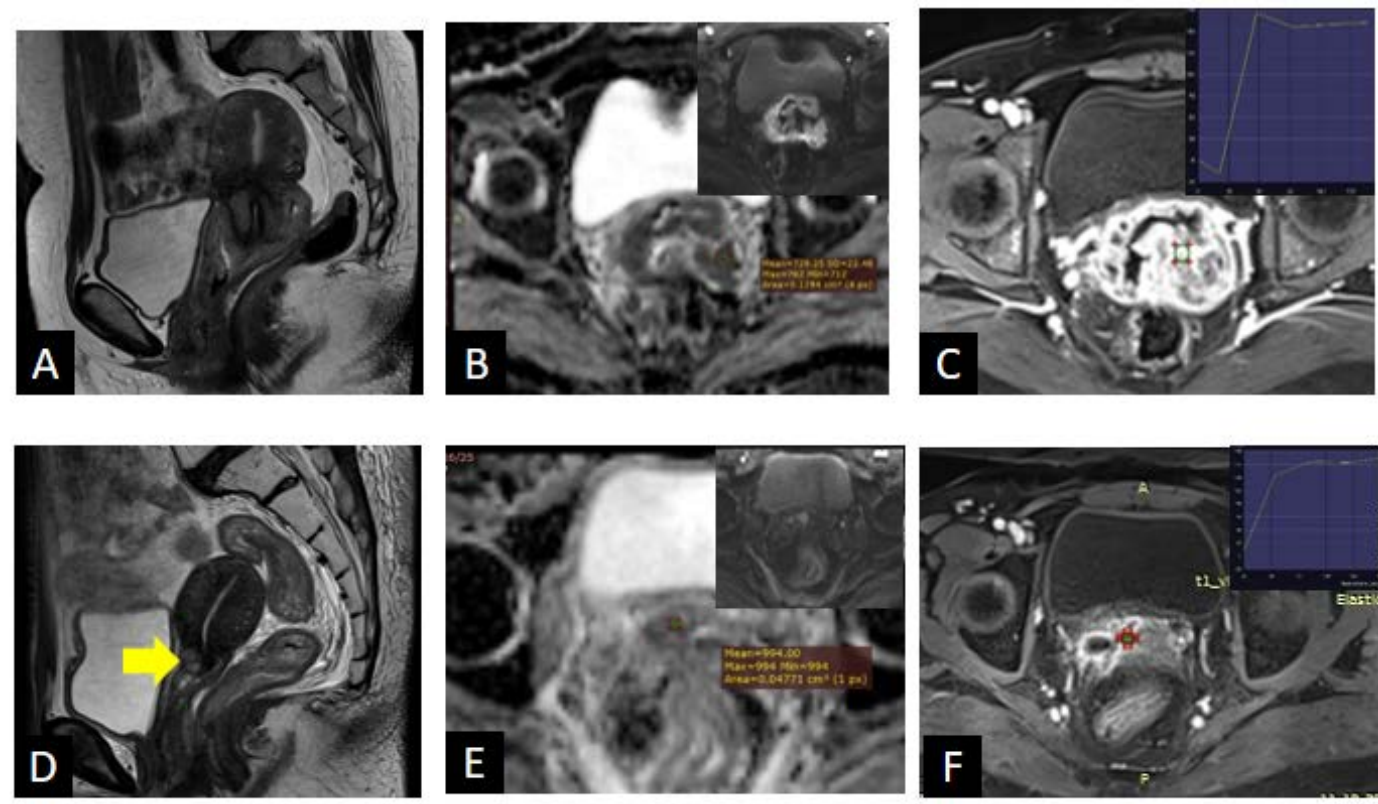

Figure 5: Baseline and Post chemoradiotherapy MRI

Baseline (A-C): T2W sagittal (A) image show infiltrating hyperintense lesion involving cervix and upper vagina. The lesion shows diffusion restriction(B) with ADC value of $0.73 \times 10^{-3} \mathrm{~mm}^{2} / \mathrm{s}$ and showing plateau pattern enhancement(C).

Post CTRT MRI (D-F): T2W sagittal (D) image show residual lesion in cervix (arrow). The lesion shows diffusion restriction with mean ADC value of $0.99 \times$ $10^{-3} \mathrm{~mm}^{2} / \mathrm{s}(\mathrm{E})$. Axial DCE image (F) shows rim enhancement with TIC depicts plateau pattern of enhancement.

\section{STATISTICAL METHODS}

The collected data were transformed into variables, coded and entered in Microsoft Excel. Data were analyzed and statistically evaluated using SPSS-PC-17 version.

Quantitative data was expressed in mean, standard deviation and the difference between two comparable groups were tested by student's t-test (unpaired) or Mann Whitney ' $U$ ' test, while qualitative data were expressed in percentage. Pre and post treatment groups were compared by Wilcoxon Signed Rank test to assess the response to treatment. 'p' value less than 0.05 was considered statistically significant.

The receiver operating characteristic (ROC) curve analysis was performed to assess the diagnostic performance. Sensitivity, specificity, PPV and NPV were calculated. Correlation ( $\mathrm{R}$ value) was determined by Spearman's Rank correlation. 


\section{RESULTS}

Conventional MR imaging was used to assess the size of tumor, signal characteristics, its extent, lymph node enlargement, and other associated findings such as pyometra, free fluid or any metastasis. On T1 W images cervical carcinoma appeared hypointense in 16 out of 21 patients (76.2 \%) and isointense in the remaining 5 patients (23.8\%). On T2 W images cervical carcinoma appeared hyperintense in all the patients. Of the total 21 patients, 10 (47.61 \%) were FIGO stage IIB cervical carcinoma, 2 patients were
(9.52 \%) stage IIIA, 4 (19.04 \%) were stage IIIB and 5 were stage IIIC1 (23.80\%). Diffusion restriction was noted in all patients with mean ADC values on baseline MRI was $0.82 \pm 0.1 \times 10^{-3} \mathrm{~mm}^{2} / \mathrm{s}$.

On post chemotherapy MRI also all patients showed restricted diffusion and mean ADC value increased by $0.14 \times 10^{-3}$ $\mathrm{mm}^{2} / \mathrm{s}$ to $0.96 \pm 0.06 \times 10^{-3} \mathrm{~mm}^{2} / \mathrm{s}$ from baseline MRI. Mean ADC value increased by $0.43 \times 10^{-3} \mathrm{~mm}^{2} / \mathrm{s}$ to $1.25 \pm 0.18 \times 10^{-3}$ $\mathrm{mm}^{2} / \mathrm{s}$ on post CTRT MRI from baseline MRI (Table 1).

Table 1: Comparison of ADC value on baseline MRI with post chemotherapy and post CTRT MRI (n= 21)
\begin{tabular}{|l|l|l|l|l|l|l|}
\hline & 1st MRI (Baseline) & $2^{\text {nd }}$ MRI (Post chemotherapy) & \multicolumn{3}{|c|}{ 3rd MRI (Post CTRT) } \\
\hline & Mean & SD & Mean & SD & Mean & SD \\
\hline ADC value $\left(\mathrm{x} 10^{-3} \mathrm{~mm}^{2} / \mathrm{s}\right)$ & 0.82 & 0.10 & 0.96 & 0.06 & 1.25 & 0.18 \\
\hline
\end{tabular}

In 3 of patients in whom residual tumor was seen on post CTRT MRI, showed $0.09 \times 10^{-3} \mathrm{~mm}^{2} / \mathrm{s}$ increase in mean ADC value on post chemotherapy MRI from baseline MRI compared to $0.15 \times 10^{-3}$ $\mathrm{mm}^{2} / \mathrm{s}$ increase in mean ADC value in patients with complete response $(n=18)$. (Table.2).

Table 2: Comparison of ADC values in patients of cervical carcinoma showing residual tumor with patients showing complete resolution on MR imaging

\begin{tabular}{|c|c|c|c|c|}
\hline \multirow[t]{2}{*}{$\begin{array}{l}\text { Residual } \\
\text { tumor }\end{array}$} & \multirow[t]{2}{*}{$\begin{array}{ll}\text { No. } & \text { of } \\
\text { patients }\end{array}$} & \multicolumn{3}{|c|}{ 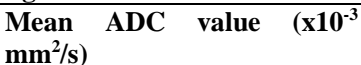 } \\
\hline & & $\begin{array}{l}\text { 1st } \\
\text { MRI }\end{array}$ & $\begin{array}{l}\text { 2nd } \\
\text { MRI }\end{array}$ & $\begin{array}{l}\text { 3rd } \\
\text { MRI }\end{array}$ \\
\hline Present & $3(14.29 \%)$ & 0.86 & 0.95 & 1.01 \\
\hline Absent & $18(85.71 \%)$ & 0.81 & 0.96 & 1.31 \\
\hline
\end{tabular}

On post CTRT MRI, complete resolution of the tumor was noted in 18 out of 21 patients (85.71\%) with an increase in mean ADC value by $0.50 \times 10^{-3} \mathrm{~mm}^{2} / \mathrm{s}$ from baseline MRI (from $0.81 \times 10^{-3} \mathrm{~mm}^{2} / \mathrm{s}$ to $\left.1.31 \times 10^{-3} \mathrm{~mm}^{2} / \mathrm{s}\right)$. Residual tumor was seen in 3 patients $(14.29 \%)$ with $0.15 \times 10^{-3}$ $\mathrm{mm}^{2} / \mathrm{s}$ increase in mean ADC value (from $0.86 \times 10^{-3} \mathrm{~mm}^{2} / \mathrm{s}$ to $\left.1.01 \times 10^{-3} \mathrm{~mm}^{2} / \mathrm{s}\right)$. Patients with complete resolution of tumor showed significant increase in mean ADC value on post CTRT MRI compared to patients with residual tumor (Table. 2) which was found to be statistically significant $(p<0.001)$.
T2 hyperintensity was noted in all 3 patients with residual tumor with diffusion restriction in the same region of $\mathrm{T} 2$ hyperintensity. But 2 patients with complete resolution of tumor also showed areas of T2 hyperintensity within cervix with no evidence of diffusion restriction and ADC values similar to other patients with complete resolution indicating that observed T2 hyperintensity was due to post radiotherapy changes rather than residual tumor.

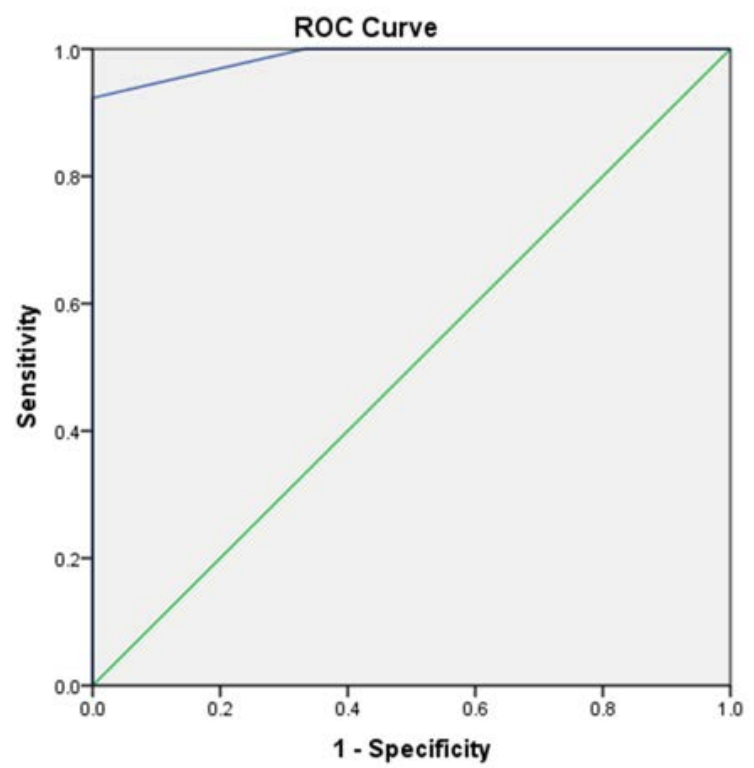

Figure 6. The ROC curve of ADC values in assessing the efficiency of chemoradiotherapy (area under the curve $0.98, p$ $<0.01$ ) 

response evaluation.

The receiver operating characteristic (ROC) curve analysis was done based on our observations. In our study when the ADC threshold value was $1.15 \times 10^{-3}$ $\mathrm{mm}^{2} / \mathrm{s}$, we could distinguish between residual tumor tissue and tumor free cervical tissue after chemoradiotherapy, with a sensitivity of $92.3 \%$ and specificity of 100 $\%$ and the area under the curve was 0.98 (Fig. 6).

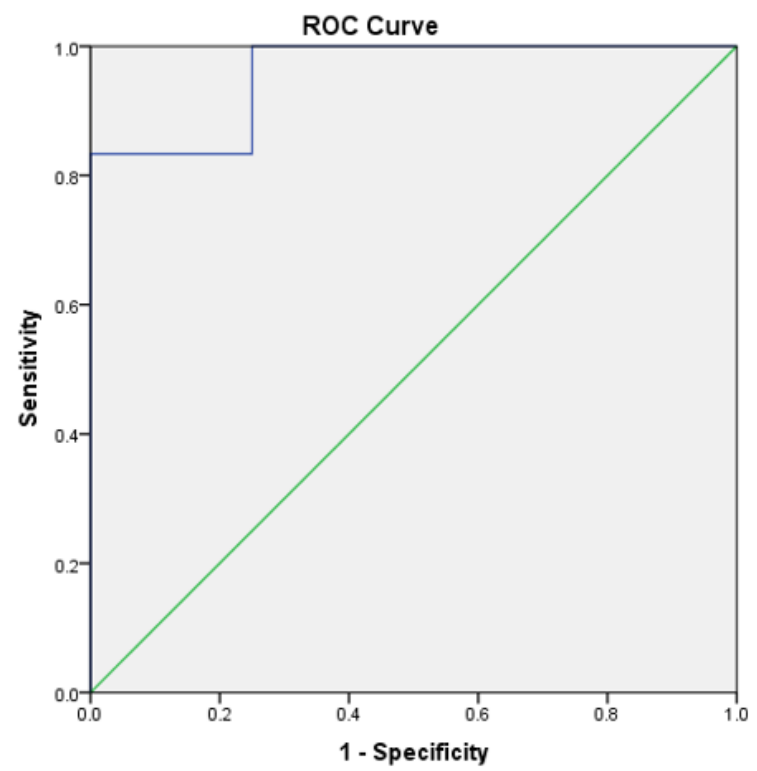

Fig 7: The receiver operating characteristic (ROC) curve analysis to assess diagnostic performance of $\triangle \mathrm{ADC}$ (increase in mean ADC value)

Diagnostic value of increase in mean ADC value in differentiating patients with complete resolution from patients with residual disease was analysed using receiver operating characteristic (ROC) curve. When an increase in mean ADC value of $0.19 \mathrm{x}$ $10^{-3} \mathrm{~mm}^{2} / \mathrm{s}$ was taken as the cut-off value to differentiate patients with complete response from patients with residual disease after chemoradiotherapy, the sensitivity and specificity were $100 \%$ and $75 \%$ respectively and area under the curve was 0.95 (Fig. 7).

Majority of the cervical carcinoma patients (17 out of 21) showed washout pattern of enhancement on baseline MRI and 4 patients showed plateau pattern of enhancement on dynamic contrast enhanced MR imaging. On post chemotherapy MRI, 9 out of 21 patients showed plateau pattern of enhancement and remaining 12 patients washout pattern of enhancement. On post CTRT MRI, 17 of the 18 patients with complete resolution of tumor showed increasing trend of enhancement and one showed plateau pattern of enhancement. None of the patients showed washout pattern of enhancement. In 3 patients with residual tumor, one patient showed plateau pattern of enhancement and 2 patients showed wash out pattern of enhancement on post CTRT MRI. None of the patients showed increasing trend of enhancement (Table 3).

Table 3: TIC curves in patients $(n=21)$

\begin{tabular}{|l|l|l|l|l|l|l|}
\hline \multirow{2}{*}{$\begin{array}{l}\text { Pattern } \\
\text { enhancement }\end{array}$} & \multicolumn{2}{|l|}{ Tst MRI (Baseline) } & 2nd MRI (Post CTH) & 3rd MRI (Post CTRT) \\
\cline { 2 - 7 } & No. of patients & No. of patients & No. of patients \\
\cline { 2 - 7 } & $\begin{array}{l}\text { Complete } \\
\text { response }\end{array}$ & $\begin{array}{l}\text { Residual } \\
\text { tumor }\end{array}$ & $\begin{array}{l}\text { Complete } \\
\text { response }\end{array}$ & $\begin{array}{l}\text { Residual } \\
\text { tumor }\end{array}$ & $\begin{array}{l}\text { Complete } \\
\text { response }\end{array}$ & $\begin{array}{l}\text { Residual } \\
\text { tumor }\end{array}$ \\
\hline Increasing trend & 0 & 0 & 0 & 0 & $17(94.45 \%)$ & 0 \\
\hline Plateau & $4(22.22 \%)$ & 0 & $8(44.44 \%)$ & $1(33.3 \%)$ & $1(5.55 \%)$ & $1(33.3 \%)$ \\
\hline Wash-out & $14(77.77 \%)$ & $3(100 \%)$ & $10(55.55 \%)$ & $2(66.6 \%)$ & 0 & $2(66.6 \%)$ \\
\hline
\end{tabular}

\section{DISCUSSION}

In tumors, changes in morphology are now known to be preceded by alterations at molecular level indicative of cellular disruption and death and are amenable to earlier detection. Studies have shown that functional imaging like DWMRI and DCE-MRI can be used to pick up these early changes at the molecular level and thereby help in predicting early therapeutic response.

We have prospectively evaluated 21 biopsy proven cases of squamous cell carcinoma of cervix considered for radical chemoradiation based on FIGO staging. Hence, the study population consisted of patients with locally advanced cervical cancer. 
DW-MR imaging studies in patients of cervical cancer has shown cancerous tissue to have significantly lower apparent diffusion coefficient (ADC) values compared to normal tissue. ${ }^{[1,16-23]}$ In our study mean ADC values of all patients on baseline MRI was $0.82 \pm 0.1 \times 10^{-3} \mathrm{~mm}^{2} / \mathrm{s}$. Our patients baseline mean ADC values were significantly lower than the mean ADC values of normal cervical tissue in control group of many studies with values ranging from 1.33 to $2.09 \times 10^{-3} \mathrm{~mm}^{2} / \mathrm{s}$. ${ }^{\text {[16- }}$ 23]

The mean ADC of cervical cancer is known to increase during and after chemoradiotherapy such that early alterations in ADC can be used for predicting early therapeutic response. In our study patients with residual tumor showed $0.09 \times 10^{-3} \mathrm{~mm}^{2} / \mathrm{s}$ increase in mean ADC values on post chemotherapy MRI from baseline MRI. Patients with complete response showed $0.15 \times 10^{-3} \mathrm{~mm}^{2} / \mathrm{s}$ increase in mean ADC values which was found to be statistically significant $(p<0.05)$. Somoye $G$ et al. conducted a study to assess prognostic values of DW-MRI and compared pretreatment ADC values with mid treatment ADC values after 2 weeks of chemoradiotherapy. They found that patients with good outcome showed greater increase in mean ADC values compared to patients with bad outcome. ${ }^{[24]}$ These early change in ADC values can be used to predict in which women the tumor would not show complete resolution show no complete resolution or subsequently recur so as to discontinue ineffective treatment and use appropriate aggressive treatment.

On post CTRT MRI, mean ADC value was $1.25 \pm 0.18 \times 10^{-3} \mathrm{~mm}^{2} / \mathrm{s}$ with increase in mean ADC value of $0.43 \times 10^{-3}$ $\mathrm{mm}^{2} / \mathrm{s}$ from baseline MRI. Many studies also showed similar increase in mean ADC values after chemoradiotherapy. ${ }^{[10,19-23]}$

After chemoradiotherapy, patients with complete resolution of tumor are known to show greater increase in mean ADC values from baseline MRI as compared to patients with residual disease.
[11,21] Our study also showed a similar result $\left(0.5 \times 10^{-3} \mathrm{~mm}^{2} / \mathrm{s}\right.$ v/s $\left.0.17 \times 10^{-3} \mathrm{~mm}^{2} / \mathrm{s}\right)$. Atstupenaitel $\mathrm{V}$ et $\mathrm{al}^{[11]}$ in their study observed that increase in mean ADC value in responders were $0.46 \pm 0.17 \times 10^{-3} \mathrm{~mm}^{2} / \mathrm{s}$ compared to $0.08 \pm 0.17 \times 10^{-3} \mathrm{~mm}^{2} / \mathrm{s}$ in non-responders after chemoradiotherapy. Study conducted by Liu $\mathrm{Y}$ et al ${ }^{[21]}$ to investigate DWI in predicting and monitoring the response of carcinoma cervix to chemoradiotherapy also showed similar observations with patients with complete response to chemotherapy showing $0.44 \times 10^{-3} \mathrm{~mm}^{2} / \mathrm{s}$ increase in mean ADC value compared to $0.27 \times 10^{-3} \mathrm{~mm}^{2} / \mathrm{s}$ in patients with partial response or static disease after chemoradiotherapy.

From our study, threshold ADC value of $\geq 1.15 \times 10^{-3} \mathrm{~mm}^{2} / \mathrm{s}$ can be used to differentiate patients with complete response from patients with residual disease after chemoradiotherapy with a sensitivity of $92.3 \%$ and specificity of $100 \%$.

DWI also helps in differentiating post radiotherapy changes from residual tumor as both these entities show increased T2 hyperintensity. Residual tumor shows diffusion restriction, where as post radiotherapy changes do not. ${ }^{[25,26]}$ In 2 of our patients who showed T2 hyperintensity in cervical region on post CTRT, MRI did not show any diffusion restriction on DWMRI confirming T2 hyperintensity was due to post radiotherapy changes rather than residual tumor.

Pattern of time-signal intensity curve (TIC) derived from dynamic contrast enhanced MR imaging can be used for differentiating cervical cancer from cancer free cervical tissue. ${ }^{[15,27]}$ Based on our study, initial uptake with washout pattern of enhancement was relatively specific for malignant lesion and progressive enhancement pattern was in favour of cancer free cervix. Plateau pattern of enhancement was also more in favour of malignancy. None of our patients showed progressive enhancement pattern on baseline MRI and 17 out of 18 patients with complete response following 
chemoradiotherapy showed progressive enhancement pattern suggesting its high specificity for cancer free cervix. To the best of our knowledge no studies have described the types of TIC in carcinoma cervix patients. Types of TIC were well established in the assessment of breast and adnexal masses with Type III TIC is specific for malignancy and type I TIC favours no malignancy. ${ }^{[27,28]}$ Type II TIC is intermediate. We got similar results in our study suggesting that types of TIC can be used for detection of malignancy in cervix. However, further studies with large study population required for the better understanding of type of TIC in assessment of cervical malignancy.

\section{CONCLUSION}

We conclude that the functional MR techniques of diffusion-weighted and dynamic contrast enhanced imaging can provide additional information regarding the tissue characteristics of cervical carcinoma. Diffusion restriction, ADC values and TIC pattern differ in patients with complete response to chemoradiotherapy from that of patients with residual tumor, hence helps in identifying patients with residual tumor.

Acknowledgment: We thank Dr. Manish Dutt, Department of Radiotherapy for his co-operation during the study.

Conflict of Interest: None

Source of Funding: None

Ethical Approval: Approved

\section{REFERENCES}

1. Bray F, Ferlay J, Soerjomataram I, Siegel RL, Torre LA, Jemal A. Global cancer statistics 2018: GLOBOCAN estimates of incidence and mortality worldwide for 36 cancers in 185 countries.CA Cancer J Clin. 2018 Nov;68(6):394-424.

2. Kundu S, Chopra S, Verma A, Mahantshetty U, Engineer R, Shrivastava SK. Functional magnetic resonance imaging in cervical cancer: current evidence and future directions. J Cancer Res Ther. 2012;8(1):11-18.

3. Susanna I. Lee, Mostafa Atri. 2018 FIGO Staging System for Uterine Cervical
Cancer: Enter Cross-sectional Imaging. Radiology. 2019; 292:15-24.

4. Balleyguier C, Sala E, Da Cunha T, Bergman A, Brkljacic B, Danza $F$ et al. Staging of uterine cervical cancer with MRI: Guidelines of the European Society of Urogenital Radiology. Eur Radiol. 2011; 21:1102-10.

5. Nicolet V, Carignan L, Bourdon F, Prosmanne O. MR imaging of cervical carcinoma: A practical staging approach. Radiographics. 2000;20:1539-49.

6. Vincens E, Balleyguier C, Rey A, Uzan C, Zareski E, Gouy S, et al. Accuracy of magnetic resonance imaging in predicting residual disease in patients treated for stage IB2/II cervical carcinoma with chemoradiation therapy: correlation of radiologic findings with surgicopathologic results. Cancer. 2008;113(8):2158-65.

7. Liyanage SH, Roberts CA, Rockall AG. MRI and PET scans for primary staging and detection of cervical cancer recurrence. J Womens Health. 2010;6(2):251-69.

8. Exner M, Kühn A, Stumpp P, Höckel M, Horn LC, Kahn T, et al. Value of diffusionweighted MRI in diagnosis of uterine cervical cancer: a prospective study evaluating the benefits of DWI compared to conventional MR sequences in a 3T environment. Acta Radiol. 2016;57(7):86977.

9. Padhani AR, Liu G, Koh DM, Chenevert TL, Thoeny HC, Takahara $\mathrm{T}$, et al. Diffusion-weighted magnetic resonance imaging as a cancer biomarker: Consensus and recommendations. Neoplasia 2009;11: 102-25.

10. Thoeny HC, Ross BD. Predicting and monitoring cancer treatment response with diffusion-weighted MRI. J Magn Reson Imaging 2010;32:2-16.

11. Atstupenaite V, Basevičius A, Krimelis A, Inčiūra A, Vaitkienè D. Diffusion-weighted magnetic resonance imaging of cervical cancer. Acta Medica Lituanica. 2011;18(4). 139-46

12. Himoto Y, Fujimoto K, Kido A, Baba T, Tanaka S, Morisawa N, et al. Pretreatment mean apparent diffusion coefficient is significantly correlated with event-free survival in patients with international federation of Gynecology and Obstetrics stage Ib to IIIb cervical cancer. Int $\mathrm{J}$ Gynecol Cancer. 2015;25(6):1079-85. 
13. Das S, Chandramohan A, Reddy JK, Mukhopadhyay S, Kumar RM, Isiah R, et al. Role of conventional and diffusion weighted MRI in predicting treatment response after low dose radiation and chemotherapy in locally advanced carcinoma cervix. Radiother Oncol. 2015;117(2):288-93.

14. Hameeduddin A, Sahdev A. Diffusionweighted imaging and dynamic contrastenhanced MRI in assessing response and recurrent disease in gynaecological malignancies. Cancer Imaging. 2015;15(1): 1-12.

15. Boss A, Massuger LF, Pop LA, Verhoef LC, Huisman HJ, Boonstra $\mathrm{H}$, et al. Post-radiotherapy contrast enhancement changes in fast dynamic MRI of cervical carcinoma. J Magn Reson Imaging. 2001;13(4):600-06.

16. Naganawa $S$, Sato Ch, Kumada H, Ishigaki T, Miura Sh, Takizawa O. Apparent diffusion coefficient in cervical cancer of the uterus: comparison with the normal uterine cervix. Eur Radiol. 2005; 15: 71-78.

17. Zhang Y, Liang BL, Gao L, Ye RX, Shen J, Zhong JL. Diffusion-weighted imaging features of normal uterine cervix and cervical carcinoma. Ai Zheng. 2007; 26(5): 508-12.

18. Xue HD, Li S, Sun F, Sun HY, Jin ZY, Yang JX et al. Clinical application of body diffusion-weighted MR imaging in the diagnosis and preoperative $\mathrm{N}$ staging of cervical cancer. Chin Med Sci J. 2008; 23(3): 133-37.

19. Charles-Edwards EM, Messiou C, Morgan VA, De Silva SS, McWhinney NA, Katesmark $\mathrm{M}$ et al. Diffusion weighted imaging in cervical cancer with an endovaginal technique: potential value for improving tumor detection in stage Ia and Ib1 disease. Radiology. 2008; 249(2): 54150.

20. McVeigh PZ, Syed AM, Milosevic M, Fyles A, Haider MA. Diffusion-weighted MRI in cervical cancer. Eur Radiol. 2008; 18: 1058-64.

21. Liu Y, Bai R, Sun H, Liu H, Wang D. Diffusion-weighted magnetic resonance imaging of uterine cervical cancer. $\mathrm{J}$ Comput Assist Tomogr. 2009; 33(6): 85862.
22. Chen J, Zhang Y, Liang B, Yang Z. The utility of diffusion- weighted MR imaging in cervical cancer. Eur J Radiol. 2010; 74(3): e101-6.

23. Chen YB, Hu CM, Chen GL, Hu D, Liao J. Staging of uterine cervical carcinoma: whole-body diffusion weighted magnetic resonance imaging. Abdom Imaging. 2010.

24. Somoye G, Harry V, Semple S, Plataniotis G, Scott N, Gilbert FJ, et al. Early diffusion weighted magnetic resonance imaging can predict survival in women with locally advanced cancer of the cervix treated with combined chemo-radiation. Eur Radiol. 2012;22(11):2319-27.

25. Makino H, Kato H, Furui T, Morishige KI, Kanematsu M. Predictive value of diffusion-weighted magnetic resonance imaging during chemoradiotherapy for uterine cervical cancer. J Obstet Gynaecol Res. 2014;40(4):1098-104.

26. Papadopouluo I, Stewart V, Barwick TD, Park WE, Soneji N, Rockall AG et al. PostRadiation Therapy Imaging Appearances in Cervical Carcinoma. RadioGraphics. 2016; 36:538-53.

27. Yang SN, Li FJ, Chen JM, Zhang G, Liao $\mathrm{YH}$, Huang TC. Kinetic curve type assessment for classification of breast lesions using dynamic contrast-enhanced MR imaging. PloS one. 2016;11(4): e0152827.

28. Malek M, Oghabian Z, Tabibian E, Rahmani M, Yazdi SN, Oghabian MA, Parviz S. Comparison of Qualitative (Time Intensity Curve Analysis), SemiQuantitative, and Quantitative Multi-Phase 3T DCE-MRI Parameters as Predictors of Malignancy in Adnexal. Asian Pacific journal of cancer prevention: APJCP. 2019;20(6):1603.

How to cite this article: Raghavendra $\mathrm{H} \mathrm{K}$, Manchanda A, Garg A et.al. Diffusion weighted and dynamic contrast enhanced magnetic resonance imaging in carcinoma of cervix: role of apparent diffusion coefficient value and time intensity curve pattern in chemoradiotherapeutic response evaluation. International Journal of Research and Review. 2022; 9(1): 17-27. DOI: https://doi.org/10.52403/ijrr.20220103 